\title{
Embraced Separation: Exploring methods of breath attunement in speculative infant swings
}

\author{
Olaoluwa Oyedokun \\ Illinois State University \\ USA \\ ooyedok@ilstu.edu
}

\author{
Kristin Carlson \\ Illinois State University \\ USA \\ kacarl1@ilstu.edu
}

\author{
Annie Sungkajun \\ Illinois State University \\ USA \\ asungka@ilstu.edu
}

\begin{abstract}
This paper explores a speculative design for an infant swing based on breath attunement. There are challenges for working parents to juggle, such as working from home and taking care of an infant, especially when infants want to be physically close to the parent. This design explores how a swing can be adaptable to subtle, dynamic changes in the parent that can soothe the infant while allowing the parent to be more hands-free. These dynamic changes include the use of breath sensors on the parent to change the rhythm of the swing, while also creating shifting amplifications of environmental sounds, as though they were being carried by the parent through the home. This work particularly explores qualities of movement and rhythm as key components of comfort for infants in their parent's arms. Results of this paper include design sketches and a usability evaluation of a smartphone interface, as a speculative approach to designing embodied interactions between parent-child relationships.
\end{abstract}

Human computer interaction. Speculative design. Healthcare. Human-centered design.

\section{INTRODUCTION}

One of the challenges of working parents with infants is keeping their infants calm while working from home. When infants hear their parent's voice, notice their presence, or lack thereof - many want to be constantly held. Since the outbreak of the COVID-19 pandemic, larger percentages of offices have been working from home and the number of remote jobs may increase due to the smooth transition of most offices not needing to operate physically, and may opt to do more online. As the world is evolving towards smart gadgets and more connectivity, the goal of designing smart technologies for home use is to improve the quality of work-life balance by using human-computer interaction concepts.

Many infant-focused commercial products focus on one-way interaction, such as the parent turning on a switch to make a swing move. Or to watch the infant through a baby monitor. Or in smart gadgets, such as the Owlet sensor, to have security that the baby has appropriate oxygen levels and is still breathing (Owlet 2017). However, it has been found that while parent-child relationships often direct attention to the parent as agents of socializations, caregiving is a two-way interaction.
Infants are biologically prepared to engage in and expect attuned interactions with caregivers (Johnson et al. 1991). This has prompted us to design a system that supports two-way interaction between infants and parents to create a secure and comforting bond over a short distance, allowing the parent to work hands free.

This paper focuses on the design of a smart baby swing that uses breath attunement and sensorimotor synchronization (SMS), rather than the typical even phrasing of motion in most commercial baby swings. SMS is the coordination of rhythmic movement with an external rhythm and is commonly studied in adults and in children (Provasi et al. 2014). Provasi et al.'s work shows that the interaction between movement and sound is present at birth. SMS is a critical part of human communication; it enables a demonstrative communication from one person and the other person responds. Consequently, SMS has been studied in a large variety of contexts including, mapping rhythmic body movements like stepping, dancing, and finger tapping to the rhythm of a visual or auditory stimulus. It is also studied by mapping more complex activities like playing music in synchrony with other players and speaking or singing in response to other vocalizations. 
We use SMS to design a speculative, smart, technology-focused infant swing that changes the swinging rhythm in relation to the parent's breath. We have designed a traditional-looking infant swing, that is controlled by the parent wearing a breath sensor with a companion app. The app allows the parent to control the intensity of the movement rhythm, as well as control additional qualities such as light color for circadian rhythms, vocal recordings of parents, music, or shifting environmental sounds. The speculative swing also comes with a small, weighted blanket to simulate a touch sensation. This paper illustrates the role that attunement could play in parent-infant bonding within smart technologies. While the initial purpose of this work was to be soothing to the infant while allowing the parent to be more hands-free, through our design process we found that the attention to embodied connection may also be beneficial to the parent.

\section{RELATED WORK}

This paper is structured to explore a speculative design for an infant swing based on breath attunement with the parent. There are challenges for working parents to juggle working from home and taking care of an infant, especially when infants want to be physically close to the parent. This design explores how a swing can be adaptable to dynamic changes in the parent's breath that can simulate embodied elements of infant-parent connection.

While growing up involves developing multiple layers of understanding the physical world, the social world, and personal world (Ayers \& Robbins 2005), infants are just starting to navigate these layers. Due to this process of starting to understand the physical world, infants are closely attached to their parents through a variety of senses including sound, touch, temperature, smell, and breath.

\subsection{Attunement, infants, and breath}

The nature of a parent's embodied affective experience has a powerful influence on how they connect to their child. Due to the strong sensory experience of developing in a womb, there is already a physically attuned connection between infant and mother (Legerstee et al. 2013). These sensations of hearing the mother's voice, movement patterns, heart rate, breath, etc. all contribute to the environment that an infant is in and the shock at adjusting to the world once born.

After birth, the mood and relationship that a parent conveys non-verbally, even unintentionally, is communicated to the baby. Because the infant is listening and attempting to make sense of their new surroundings, the parent's physical and emotional state become a key source of information
(Legerstee et al. 2013). In the ever-changing world that we are presently in, both partners are occupied with work-life balance and in an attempt to strike a balance between life coupled with giving constant attention to infants is a challenge. To address this, we look to attunement strategies as a way to feel connected and together, even when multitasks with work or domestic tasks. Embodied attunement means to synchronize bodily actions such as breath and movement. As stated in Fdili Alaoui et al., attunement is "an operation in which the observer accommodates herself to another by shifting her behaviour to the situation, process, or qualities of the other" (2015). Attunement helps understand non-verbal, emotional behaviour by enabling one person to better listen and understand what the other is feeling through aligning the senses.

When focusing on childcare, being physically close is an important aspect to soothing infants and making them feel safe. According to a mother that was interviewed, "when her children is being carried and she carries them on her back they usually stop crying because of the warmth that the child could feel, also the movement of her chest while she breaths" (Balogun \& Osawe 2021). This interview in connection to our project suggests that it is the closeness of physical proximity, and the sensory information created in this closeness, that creates a secure and calming situation for the infant. Body warmth, touch, motion of the parent's movements, motion of the parent's breath, smell of the parent, and sound of their voice all contribute to a sensation of security. While it is not possible to recreate all of these variables, we selected the breath motion as the primary element to explore in terms of attunement over a distance, because it creates a two-way interaction and physical bond between parent and child.

Because mothers and young infants in a variety of cultures around the world are behaviorally attuned with one another, moreover, correspondence in their behaviors tend to be domain specific (Balogun \& Osawe 2021). The sensory data, such as rhythm of speech, emitted by parents are different by location and cultures. One way we include this in our design is to enable the parent to record themselves singing, which can be played back throughout application. Using a parent's own song and language, in their voice, will also have a different effect on an infant than pre-recorded music.

\subsection{Rhythm and movement}

Both fetuses and newborns are used to experiencing the embodied rhythms of the mother, especially breath as a nuanced and subtle constant movement that the body is in. Due to the subtle 
movements in the expansion and contraction of the chest during breathing, infants in close proximity to parents often connect the movement to a sense of attachment and attunement to their parents (Balzarotti et al. 2014). Breath is often a key sensation in work that utilizes attunement, as synchronizing breath in activities such as movement or singing (Fdili et al. 2015). This understanding of breath, attunement, and subtle motion became interesting to us in thinking about how to create an infant device that still leverages embodied elements that simulate parent-infant bonding.

Sensor-motor synchronization (SMS) is the capacity to synchronize a rhythmic motor pattern with an externally perceived rhythm and is unique to species capable of vocal learning. A property of SMS is that two people can communicate by producing a synchronized rhythm (Provasi, et al. 2014). SMS has been studied in different movement patterns: not only movement that is fixed to a particular pattern, but in the simplest of forms like stepping, dancing and finger tapping to the rhythm of a visual or auditory stimulus. Because movement is one method for infants to feel connected to the parent, it becomes a core method in keeping infants calm and happy. This is evidenced in the plethora of infant swings and bouncers on the commercial market, as well as tricks such as driving babies around in a car to put them to sleep. Because movement is rhythmic and it can be patterned to follow a particular style, exploring these patterns became of interest to us.

\subsection{Similar Systems}

A lot of research has been focused on the Quantified Self, which has dribbled into the quantified baby (Gaunt et al. 2014). But by quantifying infant experiences, there is a potential for increasing parental anxieties as parents are introduced to "continuous stream of body data" (Gaunt et al. 2014).

The Baby Lucent project explored a speculative design for a smart pacifier and bottle, which can sense and evaluate the levels of bacteria in the infant's mouth, and the nutritional value of the food they are getting (Gaunt et al. 2014). Concerns found about these products in focus groups included items around misaligned goals (it is healthy to be exposed to bacteria to build immunity), data interpretation (how do parents know that there is concern or not if looking at raw data?), and privacy concerns (should infant data be shared?). A major concern was that the parent would learn to look at the technology for information rather than the baby. Findings from this work suggest that the infant's data should be presented to the parent only at certain times, or not constantly so the parent is not overwhelmed (we imagine that how the data is presented would be very important, since a layperson parent will not be able to interpret raw data). It was also found that the data should give parents the ability to see patterns in their children, and to only give data when the parent is attending to the child (in order to not rely on the technology).

When it comes to busy parents, it is often times difficult for them to be close to their child at all times, which adds a sense of selfdisappointment/failure/insecurity. By viewing the child through the camera option or playing recorded sounds for the child, a parent can feel more connected or feel as though they have more control over their situation. Physical closeness between parent and infant is often discussed as a key element in bonding, and learning to listen to, interpret, and attune to each other (Wang et al. 2017). However it is not always possible to be physically close due to work and domestic task requirements. Wang et al. presents a deep dive into the use of the Owlet wearable, that monitors heart rate and oxygen levels in the infant. One observation from looking at the participant details, is that none of the participants put the baby in a separate room from the parent with a baby monitor. Two-thirds of the parents kept the baby on its own cot in the same room, while one-third of parents coslept. This suggests that the parent is still focused on using their own embodied senses to track how the infant is doing, and that proximity is key to accessing that data. Many parents commented on the use of the Owlet monitor as interesting supplemental data, but that it did not give enough variety of information to really inform them about anything with the baby. The parents had tried to search for patterns between oxygen levels and heart rate with sleeping, being tired, driving in the car, etc. and the results were not as clear as they hoped.

Two smart cots have been designed to calm and soothe infants. The MIT Media Lab's "smart crib" was designed to calm a crying baby in less than a minute, by using elements from being in the womb to create a safe and secure environment. The cot begins moving in response to the baby's movements, and plays white noise in relation to the baby's cries. Evaluations were shown to reduce infant crying and increase sleep compared to other cots. Another cot, the "Suima Crib" was developed at Kyuushuu University in Japan to mimic the movements of the parent's arms while carrying the baby. When the baby's cries are sensed, the crib starts to sway at the same rate as the parent's heartbeat, at a rate of 1.8 seconds.

From learning about these different systems, we design our speculative smart crib as a reciprocating 
relationship between parent and child. It is not our intention to use technology to distance a parent, but to support distance that may be inevitable (like in work-from-home parents). When it comes to busy parents, it is often difficult for them to be close to their child at all times, which can inflict selfinsecurities. In our infant swing design, a parent can utilize a camera that peers down onto the crib to view the state of their child and feel connected, despite whatever the distance. With the breath sensor, a parent can be focused on their breathing to create a steady rhythm for their child and increase self-awareness.

In the console that contains the camera, a speaker and diffused LED strip of light is also integrated for a minimalistic design. The swing itself would be triggered via the mobile application design, where the parent can calibrate and set their breathing to the swing's actuator. This concept design would connect via Bluetooth to the mobile application and breath sensor on the parent (Figure 1).

\section{APPROACH AND SPECULATIVE DESIGN}

The goals of our infant swing design choices are to foster a more embodied connection between infant and parent. While this paper focuses on the attuned rhythm of breath between infant and parent, we have also used this goal to inform a variety of aspects of the design: visual design and mechanics, light color, recorded audio options, and a weighted blanket. These aspects together can simulate a variety of sensations to support the infant's feeling of security and agency, as noted in Johnson et al.'s work on the two-way interactions of socialization and connection (1991).

We have designed an infant swing that is controlled by the parent wearing a breath sensor with a companion app. The breath sensor enables the parent's breath rhythm to control the movement rhythm of the swing. The app allows the parent to control the intensity of the movement rhythm, as well as control additional qualities such as light color, temperature, and music. The swing also comes with a small, weighted blanket to simulate a touch sensation. Users can connect the application installed to the swing through a bluetooth connection for short-range interactions.

\subsection{Breath Mapping from Parent to Infant}

Since the breath connection is processed directly from the parent to the automation in the swing, a wearable sensor will be worn by the parent when using this feature. The sensor is an elastic band that is worn around the chest, at the base of the sternum. A conductive fabric on the band measures the amount and timing of movement of the parent's chest, and is directly translated to the actuator's back-and-forth motions.

The mapping of the interaction would follow: breathing in $\rightarrow$ actuator moves in one direction/ in positive degrees, and breathing out $\rightarrow$ actuator

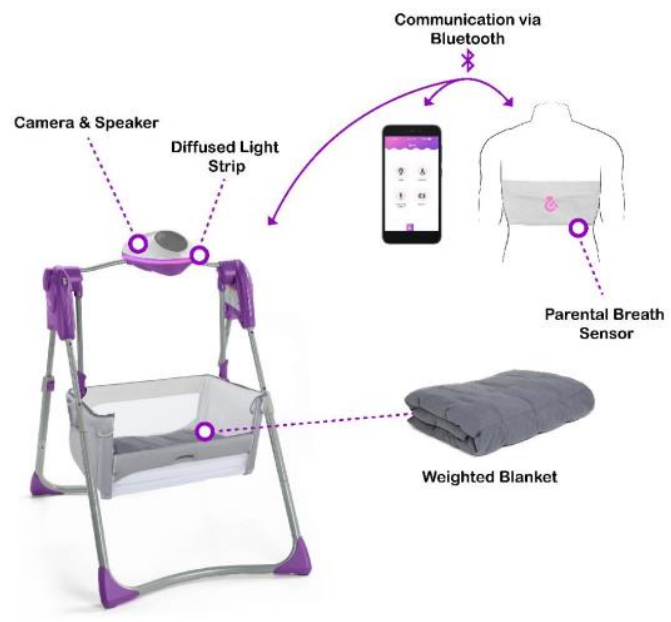

Figure 1: Concept image of baby swing design and components.

moves in the opposite direction/ in negative degrees. This mapping would already create an uneven rhythm based on how many people breathe because a breath in might be longer than breaths out. Through wizard-of-oz experiments, the authors believe that this experience of knowingly controlling the actuators of the infant swing with their breath, will make parents very aware of their use of breath and will support a meditative approach to breathing while working.

In addition to controlling the motion of the swing, the parent's use of breath brings a reflexive perspective to their own breathing. By supporting their infant by attending to themselves not only creates a bond over distance with the infant, but can support a calming strategy for the parent.

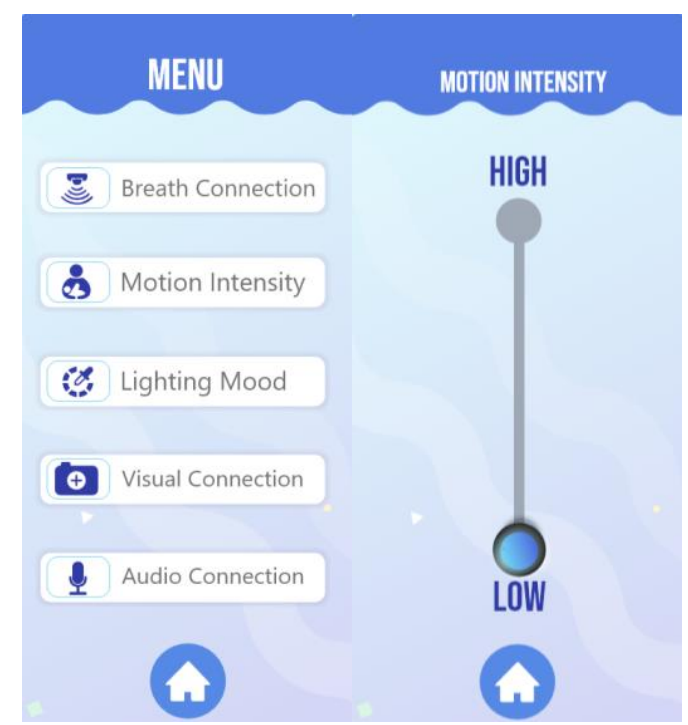


Figure 2 (left): Image of the prototype's user interface home page design.

Figure 3 (right): Image of the prototype's user interface design of rocking gauge.

This approach is reliant on the theory that if you are attending to an everyday-function in a new way (such as Heidegger's broken hammer - the tool works until it breaks (1977)), and making breath into a tool for connection with one's infant, this will bring more attention to the experience of the breath.

The app would allow the parent to control the intensity of the breath rhythm on the swing's actuator (Figure 3), in case they wanted a more vigorous swing (to help simulate a car ride to push an infant to sleep) or a more subtle swing (once the infant is asleep).

\subsection{Additional App Functions}

Additional features of this speculative design, in order to provide more variables for sensory simulation, include the ability to record and playback audio or play a simulation of environmental sounds, a weighted blanket for a tactile and warm sensation, and an embedded camera for monitoring the baby. Embedded into the console would also contain a diffused LED strip that can be controlled by the parent that supports circadian rhythms (Figure 4).

The app prototype can be found here:

https://xd.adobe.com/view/3d0a1b3d-1849-4fd88704-415aacb07b03-6859/

\subsubsection{Light Control}

This feature mainly focuses on how light can play an important role in the development of infants' circadian rhythm. Circadian rhythm refers to internally driven processes that follow a 24-hours cycle (Nigms.nih.gov). A common example of a body's circadian rhythm is its response to light and dark; during the day, we are active, but at night, we fall asleep. This is due to the brain's biological "clock", "receiving light signals from the eye, through the optic nerve" (HealthySleep), to generate a sense of alertness and therefore maintain a day-night cycle.

There is little evidence regarding how light affects an infant's sleep cycle, much less in a home environment situation (Thomas et al. 2016). However, in one study by Harrison, "babies who sleep well at night are exposed to significantly more light in the early afternoon period" (Harrison 2004), specifically 12:01-16:00. In another study, researchers measured the influence of light and maternal activity on developing infants' rhythm in
43 maternal-infant pairs (Thomas et al. 2016). It was found that infants, aged 8 and 12 weeks, showed maturation of the circadian rhythm, with light entrainment.

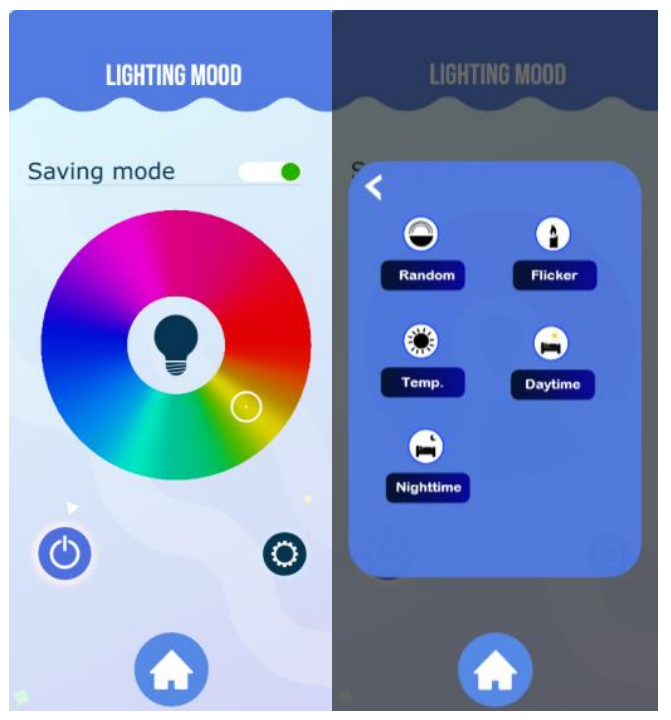

Figure 4: Image of the prototype's user interface design of light settings.

Based on the previous results of these former studies, light settings that involved color temperature and intensity were taken into consideration. By exposing the infant to a timed light that is active during the day and dim or off at night, this can potentially help promote a circadian rhythm development in babies.

\subsubsection{Record/Play Lullaby}

Voice is another important factor that creates attachments between infants and parents, in the research carried out it explains that voices constantly heard with 32-34 weeks of the baby been born registers in the subconscious mind of the babies making it surprising when babies react differently to certain voices heard or certain songs (Balzarotti et al. 2014). This supports the argument that voices are a great factor when it comes to developing connections between the parent and infant, and since the parent voice is familiar it is important to use this in our design considerations. Using a familiar voice rather than a pre-recorded unfamiliar voice can be used to help create another form of bonding over distance. Baker and Mackinlay studied the effects of mother's singing lullabies to their infants, by creating a lullaby educational program, and it resulted in a form of additional bonding, and another form of communication between mother and baby (2006). By singing to the baby and observing their response, mothers indicated that they could better understand the babies' communication. 
Our design emphasizes that parent voices can be recorded and played back to the infants to further strengthen the attachment while the infants are in the swing all alone to themselves. A stronger bond between parent and child makes a lullaby more successful in putting the child to sleep because the child is more likely to rest easy in the presence of someone they love and are bonded to. The process of singing is also important for the parent, as it engaged them in an embodied form differently than with other senses (Baker and Mackinlay, 2006).

Additionally, the application has an option for mothers to record lullabies or audio clips to be played back to their child via Bluetooth connection to the speaker on the baby swing. Lullabies are often sung by parents in order to soothe distressed children or to lull a child to sleep. As modern day mothers become busy with work-life balance, there is a preference to use pre-recorded music as a substitute for the action of singing to their babies. However, it has been shown that infants will prefer to hear their mothers' voices (Lecanuet 1996).

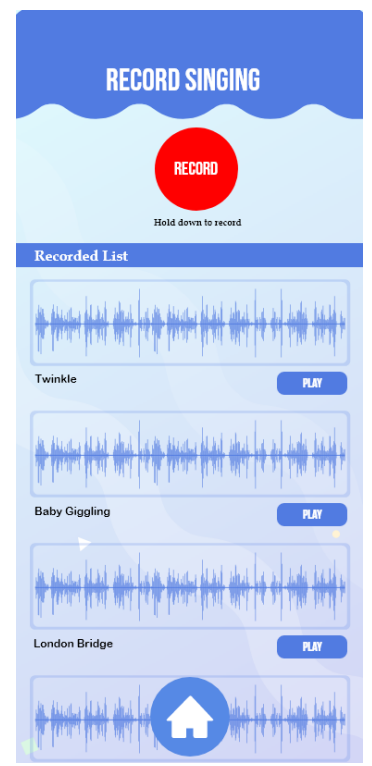

Figure 5: Image of the prototype's user interface design of recording audio settings.

By being able to record themselves singing, parents can save voice tracks to play back remotely, for when they find themselves busy or unable to sing to their child in the moment. It maintains a connection to the infant as they will recognize their parents' voice and potentially be soothed.

\subsubsection{Additional Audio}

In addition to playing the parent's lullabies, there will also be standard music options, white noise, as well as shifting environmental sounds. The concept of shifting environmental sounds is to support the concept of movement with the parent - that environmental sounds shift as one moves through the environment. Most available white noise apps include a variety of options for single-item recordings that can be switched between: the washing machine, a fan, rain. Our shifting environmental sounds would be a pre-recorded composition that includes subtle shifts in sound modeled after an acoustic ecology-focused sound walks (Westenkamp, 2021). This approach acknowledges that the sounds around us layer and combine to become a composition by the time we hear them. In future work, we are interested in whether the combination of non-even breath rhythms in the movement of the swing and an audio composition of a slow walk through different environments would support better sleep patterns. Prior research has found that using two sensory modalities in media that simulate the environment helps the audience to experience that as "real", even if the media is not real (Corness et al.2011).

\subsubsection{Sensation of Touch}

According to Balzarotti et al., emotional attunement helps human-human communication studies understand non verbal emotional behaviour (2014). This can be explored with the weighted blanket that transfers heat from parent to infants, it is understood that parent's body warmth has a sense of connection with infants. The body warmth from the parent keeps the infants calm and since it is transferable through sensors to the weighted blankets which in turn keeps the infants warm and the heat is transferred to keep the connection.

\subsection{Usability Report}

The application was designed to be minimalistic, clear, and easy to use while holding children or multitasking. This included making a clear home button that would take the user back to a central menu screen with the options of: Breath Connection, Motion Intensity, Lighting Mood, Visual Connection, and Audio Connection. Each menu item is for a sensory modality: Breath Connection is the bluetooth setup for the breath sensor, and where raw data could be viewed. Motion Intensity consists of a knob interface to shift the intensity of the rocking, based on the breath connection. Lighting Mood enables the user to make light color changes, while suggesting options to improve circadian rhythms. Visual Connection gives a direct camera view of the infant, while Audio Connection has options for both recording audio of the parent and playback, as well as playing white noise, music, or a sound-walk recording that focuses on subtle shifting environmental sounds.

In order to test the mobile application design, a plugin called Useberry for Adobe XD was used to evaluate the interface choices. An in-depth focus group that explores the embodied nature of attunement through the breath interface will be 
completed in future work. Tests were created and then shared with two participants, User A (male) and User B (female). The plugin recorded the users' clicks, length of time on tasks, and analyzed whether or not the users completed, gave up, or dropped off (meaning the user terminated the test due to inactivity or closing the window). Before the test, the participants were told different scenarios and were studied based on their decisions.

\section{User A:}

The question for this test was: "Your baby is crying in the room and you are in a short meeting. How will you check on the baby before the meeting ends?'

Steps the user carried out:

The user turned on the light.

Checked the baby through the camera.

Played songs from the recorded list.

Breakdown of the steps:

This user, having heard the voice of his baby crying in the cot, tried all possible options the application had, which made the user spend 37.3 seconds and 18 clicks. The focus of this test was to have an insight on how young parents think when they are finding a solution. When attempting to calm their toddler, it was noted that they will expend their options before they find the most optimal option that works.

After the experiment, the user was asked what their experience was and how they could relate to it. Their response revealed that the application appealed to the young father who utilized many of the options covered in the application. The recorded lullaby and playback option was found to be a viable alternative for the father, for when he is unable to sing to his child.

However, it was noted that he had a challenge with the rocking system of the swing, being controlled by the app. An inherent paranoia of the safety of his child was evident, as he feared for the possibility of his child falling off. Due to this, there was a tendency to not utilize this feature, while he was away.

\section{User B:}

The question for this test was: "The user is in a meeting and suddenly doesn't hear the giggling of her baby."

She first noted that she would likely run into the room, in order to check on the toddler, just to be reassured that they were fine.

Steps the user carried out:

Log into the app.
Easy access to the homepage.

Click on camera.

With the instinctive need to check on her toddler, this navigated the user through the application towards the camera option. Due to this option's easily accessible location in the homepage, the user had fewer clicks in her task and spent time observing her toddler. In total, the user spent 25.4 seconds and took 6 clicks to reach her goal.

Overall, the mobile application had a high rating for accessibility for its distinct icons and clear separation of colors from the background. The icons were easily recognizable and users knew where they were and where not to click. In Figure \#, a heat map shows the navigation of the users and how effective the user experience was.

\section{CONCLUSION AND FUTURE WORK}

This paper presents a speculative design of a smart baby swing that uses sensori-motor synchronization (SMS) to change the swinging rhythm in relation to the parent's breath, by wearing a breath sensor. By using a mobile application, the parent can control the intensity of the movement rhythm, along with additional features of the smart crib such as light, music, and a camera. While the initial intention of the work was to assist in soothing an infant, so parents could be more hands-free, we found that there is a potential embodied connection that may be beneficial to the parent. By emphasizing the parent's breath in the design, we brought an embodied approach that is a key element in parent-infant bonding. This interaction is bi-directional: the infant swing is moving in a rhythm using the parent's breath pattern, and the parent thus attends more to their breath. We believe this reflective choice would support bonding over a distance, as the parent is still attending to the infant by attending to themselves. Our current evaluations include self-reflections and an evaluation of the app interface design.

One potential limitation to this work is the argument in other works that suggested that using technology to understand infants could create more of a divide between parents and infants. However, as we are exploring how to create a more embodied sense of connection even while apart, we do not see this as a technology that would be used to not physically check on an infant.

In the future, we plan to develop an in-depth focus group that explores the embodied nature of attunement through the breath interface, as well as develop a functional prototype to evaluate the feasibility of this work in a real-world scenario. 


\section{REFERENCES}

Baker, F., \& Mackinlay, E. (2006) Sing, Soothe and Sleep: A Lullaby Education Programme for FirstTime Mothers. British Journal of Music Education, 23(2), 147-160.

Balogun S. and Osawe O. (2021) [Personal Communication]. Interviews via phone call. February 21, 2021.

Balzarotti, S., Piccini, L., Andreoni, G., \& Ciceri, R. (2014) "I Know That You Know How I Feel": Behavioral and Physiological Signals Demonstrate Emotional Attunement While Interacting with a Computer Simulating Emotional Intelligence. Journal of Nonverbal Behavior, 38(3), 283-299.

Bornstein, M. H. (2013) Mother-infant attunement: A multilevel approach via body, brain, and behavior. In The infant mind: Origins of the social brain, pp. 266-298. The Guilford Press.

Corness, G., Carlson, K., \& Schiphorst, T. (2011) Audience empathy: A phenomenological method for mediated performance. Proceedings of the 8th ACM Conference on Creativity and Cognition, pp. 127-136.

Fdili Alaoui, S., Schiphorst, T., Cuykendall, S., Carlson, K., Studd, K., \& Bradley, K. (2015) Strategies for Embodied Design: The Value and Challenges of Observing Movement. Proceedings of the 2015 ACM SIGCHI Conference on Creativity and Cognition, pp. 121-130.

Haft, W. L., \& Slade, A. (1989) Affect Attunement and Maternal Attachment: A Pilot Study. Infant Mental Health Journal, 10(3), pp. 157-172.

Harrison, Y. (2004) The relationship between daytime exposure to light and night-time sleep in 612-week-old infants. Journal of Sleep Research, 13: pp. 345-352.

HealthySleep (2007) Under the Brain's Control. http://healthysleep.med.harvard.edu/healthy/scienc e/how/neurophysiology (Retrieved 15 March 2021).

Heidegger, M. (1977) Basic Writings. Harper Perennial Modern Classics.

Lecanuet, J.P. (1996) 'Prenatal auditory experience', in I. Deliège \& J. Sloboda (Eds), Musical Beginnings: Origins and Development of Musical Competence, pp. 3-25. New York: Oxford University Press.

Legerstee, M., Haley, D. W., \& Bornstein, M. H. (2013) The Infant Mind: Origins of the Social Brain. Guilford Publications.
Lipari, L. (2014) Listening, Thinking, Being: Toward an Ethics of Attunement. Penn State Press.

Siegel, D. J. (2007) The Mindful Brain: Reflection and Attunement in the Cultivation of Well-Being (Norton Series on Interpersonal Neurobiology). W. W. Norton \& Company.

Liu, Y., Zheng, D., Lin, T., Liu, X., Wang, D., \& Hopfgartner, F. (2018) Smart Crib Control System Based on Sentiment Analysis. 2018 IEEE 16th Intl Conf on Dependable, Autonomic and Secure Computing, 16th Intl Conf on Pervasive Intelligence and Computing, 4th Intl Conf on Big Data Intelligence and Computing and Cyber Science and Technology Congress, pp. 222-229.

National Institute of General Medical Sciences (2021) Circadian Rhythms.

https://www.nigms.nih.gov/education/fact-

sheets/Pages/circadian-rhythms.aspx (Retrieved 15 March 2021).

Owlet. 2017. Owlet Smart Sock; Baby Care. Owlet Care. http://www.owletcare.com/ (Retrieved March 15, 2021)

Provasi, J., Anderson, D. I., \& Barbu-Roth, M. (2014). Rhythm perception, production, and synchronization during the perinatal period. Frontiers in Psychology, 5, 1048.

Quito, A. 2016. This MIT Media Lab-engineered "SMART Crib" is designed to quiet a crying baby in under a minute. https://qz.com/815858/snoo-asmart-crib-that-instantly-quiets-crying-babiesdesigned-by-harvey-karp-yves-behar-and-deb-roy/ (Retrieved March 14, 2021).

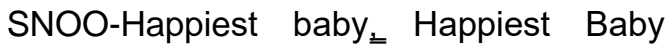
(2018) [Website] https://www.happiestbaby.com/pages/snoo. (Retrieved March 14, 2021).

Thomas, K. A., Burr, R. L., \& Spieker, S. (2016). Light and maternal influence in the entrainment of activity circadian rhythm in infants 4-12 weeks of age. Sleep and biological rhythms, 14(3), 249-255. https://doi.org/10.1007/s41105-015-0046-2

Toto, S. (2009) The Suima crib is the world's first fully automatic baby bed: https://techcrunch.com/2009/02/02/the-suima-cribis-the-worlds-first-fully-automatic-baby-bed/ February 2, 2009. (Retrieved March 15, 2021).

Westerkamp, H. (n.d.) Writings by Hildegard Westerkamp. [Website]

http://www.hildegardwesterkamp.ca/writings/writing sby/index.php (Retrieved March 17, 2021). 